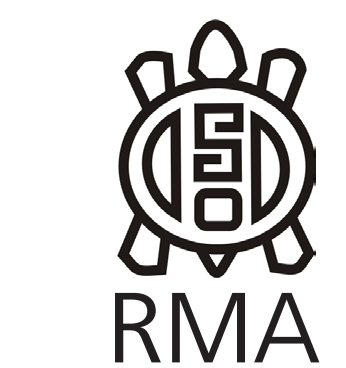

VI Jornadas

Arqueológicas Cuyanas

\title{
Canteras-taller El Ranchito (Dpto. Ischilín, Córdoba). Una primera aproximación a la caracterización de las fuentes y al análisis de los conjuntos líticos
}

\author{
Quarry-workshop El Ranchito (Dpto, Ischilín, Córdoba). A \\ first approach to characterize provenience sources and \\ the analysis of lithic sets
}

\author{
Gisela Sario*, Eduardo Pautassi* y Marcos Salvatore**
}

*IDACOR-CONICET y Museo de Antropología, Facultad de Filosofía y Humanidades, Universidad Nacional de Córdoba, Argentina. E-mail: giselasario@hotmail.com; e_pautassi@yahoo.com.ar ${ }^{*}$ CNEA (Comisión Nacional de Energía Atómica, Regional Centro), Córdoba, Argentina.

E-mail: marcossalvatore@hotmail.com

\begin{abstract}
Resumen
El objetivo de este trabajo es presentar una primera aproximación a la caracterización de los afloramientos y el análisis tecno-tipológico de materiales líticos recolectados en una cantera-taller, ubicada en la localidad arqueológica El Ranchito (valle de Copacabana, Dpto. Ischilín), en el norte de la provincia de Córdoba. A lo largo de las investigaciones arqueológicas en el valle se menciona la presencia de una materia prima lítica de muy buena calidad para la talla, una roca silícea, de la cual se han hallado puntas de proyectil y otros artefactos formatizados. Si bien su presencia es escasa en comparación con otras materias primas como el cuarzo, la misma se encuentra en la mayoría de los sitios arqueológicos de la región. En la localidad El Ranchito se han identificado tres fuentes primarias de aprovisionamiento de este material, como resultado de cuatro prospecciones en donde se realizaron ocho cuadrículas de recolección de material lítico en diferentes sectores. Los relevamientos permitieron establecer la disponibilidad natural de esta materia prima, extrayéndose muestras de la fuente identificada que fueron inspeccionadas con microscopio e identificadas como silcrete. Aquí se presentan los resultados del análisis de la primera unidad de recolección realizada, en la que se recuperaron en su mayoría ecofactos y desechos de talla, y en menor proporción núcleos e instrumentos. Esto nos permite inferir que la actividad principal era el descortezamiento de nódulos.
\end{abstract}

Palabras clave: cantera-taller; silcrete; afloramientos; análisis tecno-tipológico; Córdoba

\begin{abstract}
This paper aims at presenting a first approach to the characterization of outcrops and techno-typological analysis of lithic materials collected in a quarry-workshop, located in the archaeological site El Ranchito (Valle de Copacabana, District of Ischilín) in the northern province of Córdoba. During archaeological research in the valley, we found lithic raw material of high quality for carving, a siliceous rock from which arrowheads were identified, in addition toother stone tools. While its presence is low as compared with other lithic artifacts such as quartz, such material was found in most archaeological sites in the region. In El Ranchito, three sources of this material were identified, in which quarryworkshop areas were formed. These resulted from four surveys in which eight collection grids of lithic material were conducted in different areas. The surveys helped establish the natural availability of this raw material, through which samples were analyzed under microscope and identified as silcrete. We report the results from the analysis of the first collection unit, in which we found, mostly, ecofacts and debitage, and, to a lesser extent, cores and tools, allowing us to infer that tasks related to the removal of nodule cortex were performed.
\end{abstract}

Keywords: quarry-workshop; silcrete; outcrops; techno-typological analysis; Córdoba

La arqueología del valle de Copacabana y sus áreas adyacentes ha sido tema de interés para distintos investigadores desde los primeros trabajos de R. González (1956-1958) en la década de 1950. Particularmente en la localidad arqueológica El Ranchito se realizaron investigaciones durante la década de 1970 por Marcelino (2001), quien aborda específicamente el estudio de restos humanos fechados por el método de radiocarbono en ca. 
3000 AP. También menciona el hallazgo de una "cantera de ópalo" en los alrededores y la presencia de objetos líticos. Posteriormente, Laguens y equipo (Laguens 1999) detectan 14 concentraciones. En una de ellas se efectuó una excavación sistemática en la que se recuperaron mayoritariamente materiales líticos, y en menor cantidad cerámicos y faunísticos. En cuanto a la cronología se realizó un fechado radiocarbónico a una estructura subterránea de tierra cocida cuyo resultado fue de ca. 370 años AP, lo que demuestra la ocupación del lugar hasta épocas de contacto hispano-indígena.

Por otra parte, con el aporte interdisciplinario, se realizaron una serie de análisis de los procesos de formación naturales que afectan el registro arqueológico (Laguens 1993-1994), y que constituye uno de los primeros trabajos en esta temática para la provincia de Córdoba.

En el año 2012 se retomaron distintos aspectos de la tecnología lítica y particularmente se enfocaron los estudios en construir una base regional de recursos líticos para la región. Actualmente hay un incremento en las investigaciones acerca de la utilización prehispánica de fuentes de materias primas en el norte de Córdoba centradas principalmente en estudios tecno-tipológicos y no tipológicos sobre el cuarzo (Caminoa 2014; Cattáneo y Caminoa 2015; Sario 2013a; Sario y Pautassi 2015; Sario et al. 2015).

En el caso del resto de las rocas silíceas, no se contaba con estudios específicos, por lo que se comenzó recientemente con la caracterización de los afloramientos y de muestras de esta materia prima en particular. Investigaciones similares se han realizado con muestras arqueológicas de sitios procedentes del sur de San Luis (Heider 2015), y con muestras geológicas y arqueológicas del noreste de San Luis (Sario 2011, 2013b). Dichos estudios han permitido caracterizar las fuentes y disponibilidad de materias primas utilizadas por los diferentes grupos humanos que habitaron dichas regiones.

El objetivo de este trabajo es dar a conocer los primeros resultados de los estudios aplicados a estas rocas silíceas, con mapeos de superficie, la descripción macroscópica, microscópica y el análisis lítico.

\section{El Ranchito}

La localidad arqueológica El Ranchito (Dpto. Ischilín, provincia de Córdoba), se encuentra próximo al poblado de Chuña, en un área de llanura en la que finalizan las Sierras Chicas. El curso de agua principal es el río Copacabana, que corre de sur a norte y se insume en la depresión de las Salinas Grandes. En esta localidad se hallan varios sitios a cielo abierto ubicados en las márgenes del Arroyo 28, afluente del río Copacabana, en donde se encuentra una distribución continua de material arqueológico en superficie.

\section{Los afloramientos de rocas silíceas y los sitios cantera-taller}

Para comenzar a abordar el estudio de estas fuentes, se realizó una prospección intensiva del tipo estratificada aleatoria (Cerrato Casado 2011) buscando identificar los sectores donde la materia prima estuviera disponible naturalmente. Estas rocas se hallan constituyendo silcretes en niveles de sedimentos finos castaños amarillentos ricos en calcretes. El silcrete es un término utilizado para describir las acumulaciones y/o reemplazo producto de procesos superficiales de sílice en suelos, sedimentos o material meteorizado, para formar una duricostra (Nash y McLaren 2007). Los mismos se ubican por debajo de los niveles limosos que contienen el material arqueológico (Fm Guanaco Muerto) (Candiani et al. 2001; Carignano 1997) (Figura 1). Los niveles ricos en toscas y en rocas silíceas constituyen pequeñas lomadas debido a que son más resistentes a la meteorización. Se observan como costras de forma irregular con nódulos de sílice de dimensiones variables, siendo más frecuentes los de 2 a $15 \mathrm{~cm}$, que presentan un débil bandeamiento. Los nódulos menores a $10 \mathrm{~cm}$ presentan un mayor grado de homogeneidad (sin inclusiones, oquedades o corteza) con respecto a los nódulos de mayor tamaño.

Se han registrado tres áreas donde aflora esta materia prima (Figura 2a), dos de estas corresponden al sitio cantera-taller El Ranchito 1, y tienen una superficie de $0,144 \mathrm{Ha}$, y la tercera, corresponde al sitio cantera-taller El Ranchito 2, con una superficie de 0,414 Ha. Para su descripción macroscópica se tomaron distintas muestras de los afloramientos y de piezas arqueológicas. Se seleccionaron un total de diez muestras procedentes de los tres afloramientos, dos de nódulos sueltos y ocho de extracciones de nódulos cementados, estos últimos aprovechando la disolución del material calcáreo para la obtención de muestras lo más homogéneas posibles. Las muestras arqueológicas son cuatro y corresponden a instrumentos formatizados.

Por otra parte, se realizaron ocho cuadrículas de recolección en sectores inmediatos a las fuentes, de 1 , 2 y $10 \mathrm{~m}^{2}$. La primera, cuyos resultados presentamos aquí, es de $1 \mathrm{~m}^{2}$ y estuvo ubicada en el sitio 1 (unidad de recolección 1) en un sector de pendiente plana y de concentración de material (Figura 1). Se procedió a levantar todo el material, incluso aquellos que se encontraban semi-enterrados llegando a una profundidad de aproximadamente $20 \mathrm{~mm}$. Las siete cuadrículas restantes fueron realizadas cuatro en el sitio 1 y las otras tres en el sitio 2 .

\section{Caracterización macroscópica}

Las muestras están representadas por material de color gris blanquecino, gris claro y traslúcido de aspecto opalino, con una dureza entre 6 y 7 (escala de Mohs) con 
Figura 1. Mapa del área de estudio

Figure 1. Map of study area

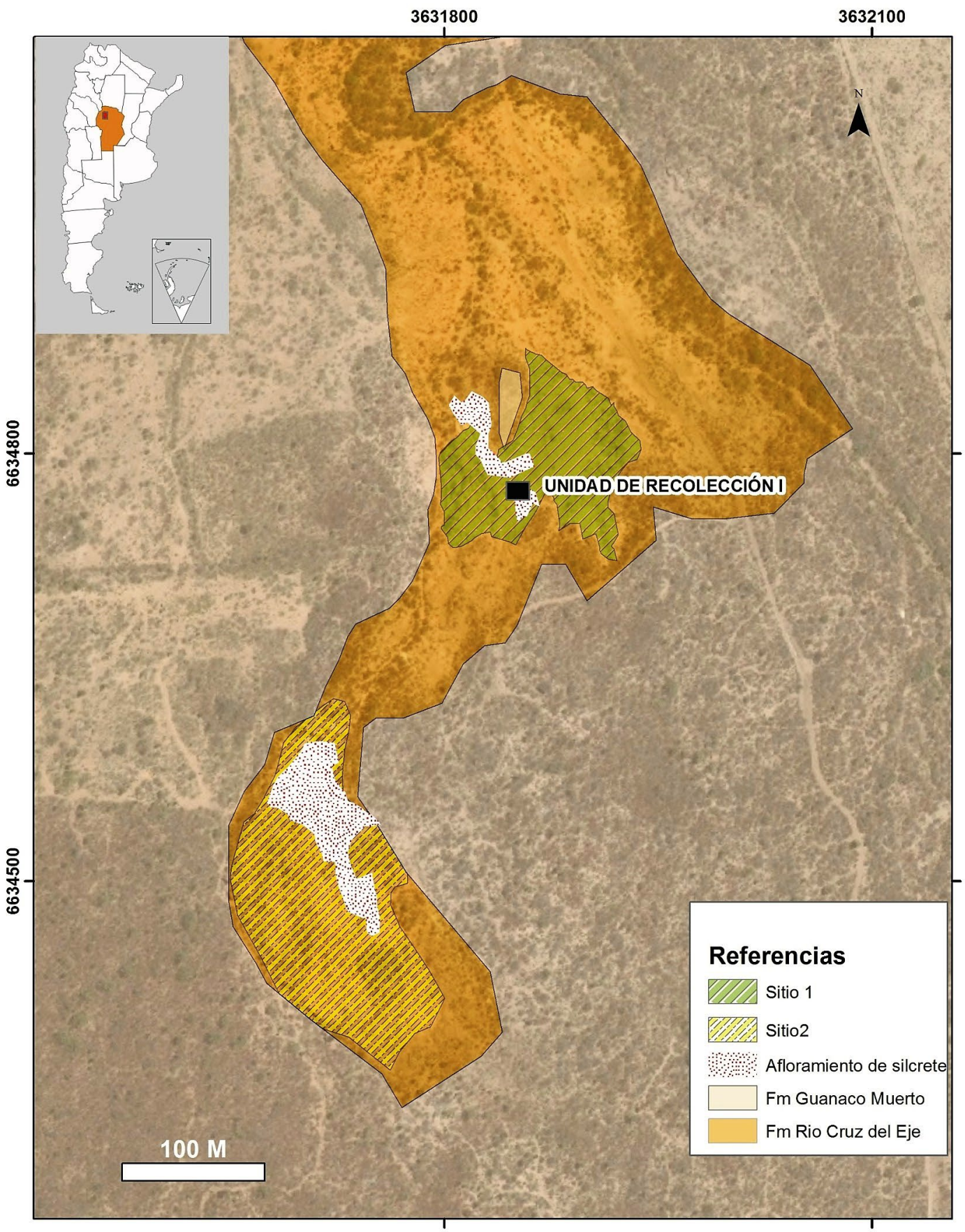

fractura concoidea (Figura 2b, c, d). Además presenta inclusiones de limos de color castaño amarillento y abundantes oquedades tapizadas por bandas de sílice opalino de color gris blanquecino. También presentan una textura bandeada, con niveles más ricos en limos y otros constituidos exclusivamente por sílice (Figura 2). Se realizaron experiencias de talla con esta materia prima y mostró ser de muy buena calidad.

\section{Caracterización microscópica}

La muestra está compuesta por un $80 \%$ de cristales esferulíticos de Quartzine (calcedonia largo-lento), con un tamaño de esferulitas de entre $200 \mu \mathrm{m}$ y $10 \mu \mathrm{m}$ siendo los más abundantes 50 y $150 \mu \mathrm{m}$ y también agregados fibrosos largo lento rellenando microfisuras (Figura 2e, f). En menor proporción se presenta micro cuarzo (Flörke 1991) tipo puzzle de tamaño de grano $>10 \mu \mathrm{m}$ con formas ameboidales y bordes intercrecidos con otros cristales de cuarzo de grano muy fino, con extinción ondulosa. También se observan escasos cristales de cuarzo euhédricos que se presentan zoneados mostrando sobrecrecimiento a partir de granos de cuarzo detríticos del sedimento o sobre cristales de microcuarzo. De forma aislada se encuentran óxidos de hierro.

\section{Análisis lítico}

En el sitio cantera taller El Ranchito 1 se realizó la recolección superficial del material de la cuadricula 1, 

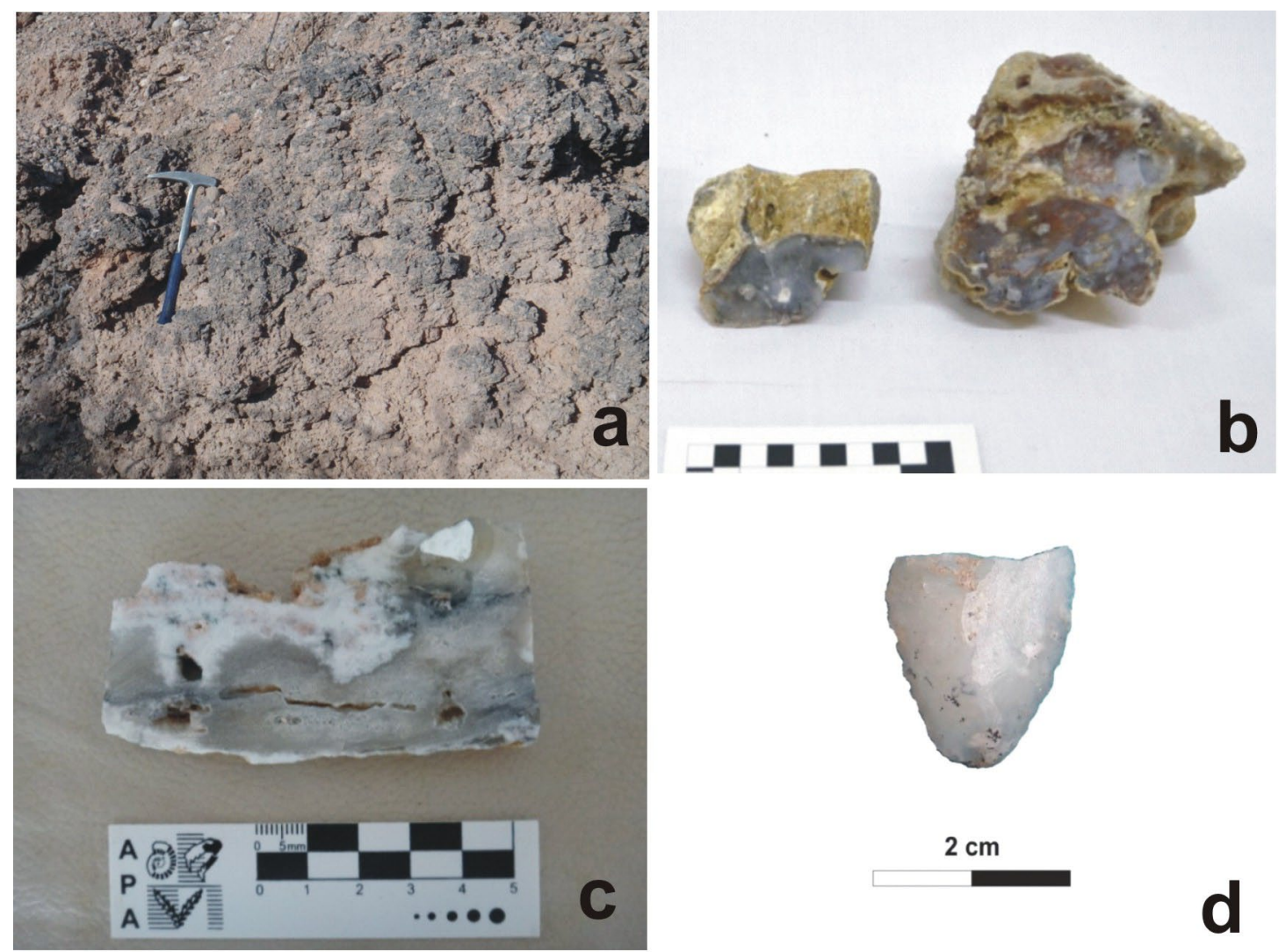

$2 \mathrm{~cm}$
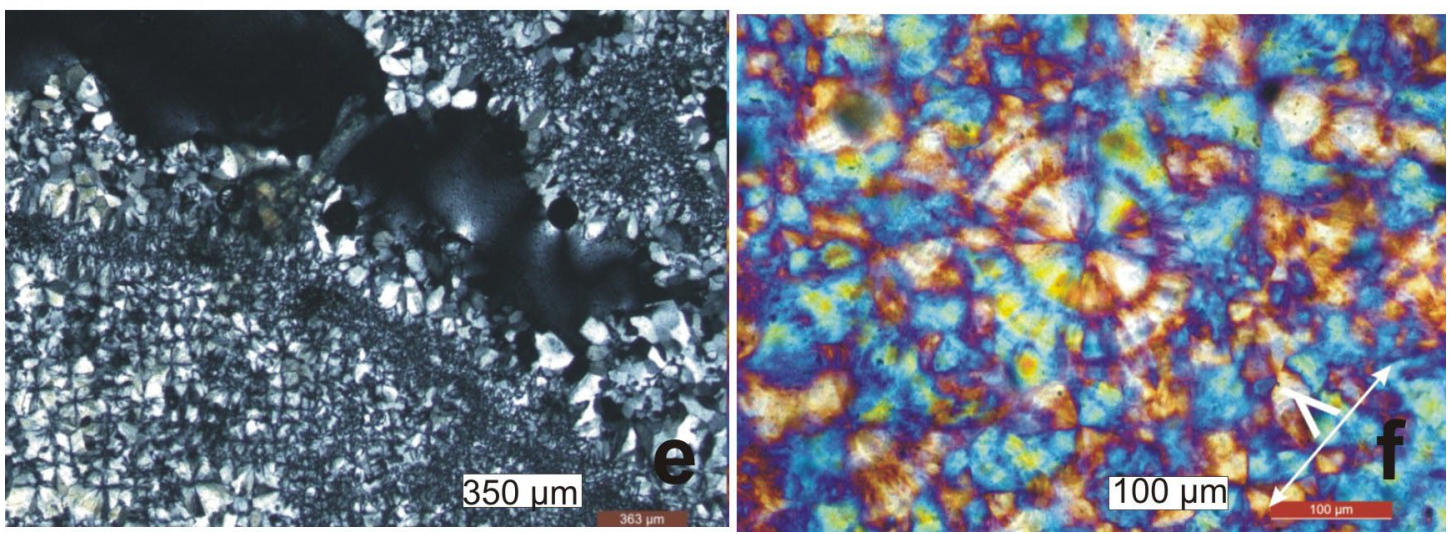

Figura 2. a: afloramiento, b-c: muestras de mano, d: pieza arqueológica, e-f: cortes delgados.

Figure 2. a: silcrete outcrops, b-c: hands specimen, d: archaeological tool, e-f: thin slices

pudiéndose recuperar 396 piezas. Las variables que se utilizaron para abordar el estudio de este material, siguiendo la propuesta de Aschero (1975, 1983), fueron: materia prima, medidas longitudinales, clase artefactual, presencia o ausencia de corteza, tamaño, forma geométrica. Para el caso de lascas se tomaron además los atributos como el tipo de talón, tipo de lasca y cantidad de negativos en la cara dorsal, y en el caso de instrumentos se midieron la longitud, el ancho de los filos, los ángulos y los retoques. Criterios propios fueron sumados a esta clasificación para la forma geométrica, modificando la ya establecida por Aschero (op cit.), y para el conteo de superficies sin corteza.

La muestra recuperada en El Ranchito sitio 1 está compuesta por un $72 \%$ de artefactos $(\mathrm{N}=285)$ y un $28 \%$ $(\mathrm{N}=111)$ de ecofactos. Los artefactos son en su totalidad de silcrete, a excepción de un desecho de riolita de muy buena calidad para la talla. Las clases artefactuales más representadas son los desechos de talla indiferenciados, seguidos por las lascas, filos naturales con rastros complementarios, instrumentos retocados, y núcleos (Tabla 1). En el caso de los instrumentos retocados, tecnomorfológicamente son raspadores. Los artefactos con corteza son 271 (95\%), y solo 14 no presentan corteza. Con respecto a la forma geométrica de los artefactos 141 (49\%) poseen formas planas, tabulares o laminares. En cuanto a los tamaños de los desechos y lascas hay un $2,11 \%$ de tamaño muy pequeño, $43,88 \%$ del pequeño, $35,86 \%$ de mediano-pequeño, $13,08 \%$ de mediano- 
grande, $2,95 \%$ de grande y $2,11 \%$ del muy grande.

\section{Discusión}

Varios autores han descripto una materia prima similar como calcedonia (Austral y Rochietti 2004; Cattáneo 1994; González 1960; Heider 2015; Laguens y Bonnin 1987; Rivero y Pastor 2004). En nuestro caso de estudio, la fuente de materia prima aquí presentada resulta por sus características macroscópicas similar a aquellas halladas en otros sitios arqueológicos, pero la descripción del contexto geológico y la petrografía han permitido caracterizarla como un silcrete. Resultó de utilidad además de la caracterización petrográfica de la muestra arqueológica conocer de manera detallada el contexto geológico para determinar el tipo litológico de esta materia prima. La incorporación de nuevos análisis de materias primas similares permitirá correlacionar esta roca con otros sitios, pudiendo establecer si se trata de la misma roca y plantear hipótesis sobre su transporte o circulación en distintas regiones.

Los resultados preliminares del análisis lítico de esta primera muestra recolectada, están indicando que los primeros momentos de los procesos de reducción son los más frecuentes en el lugar, y que probablemente las formas base se estén trasladando a otros sitios. La presencia de una gran cantidad de ecofactos (en su mayoría toscas), de desechos indiferenciados y artefactos con corteza nos permite inferir que se estaban realizando actividades de descortezamiento de nódulos. El hallazgo de algunos instrumentos, probablemente se relacione con el desarrollo de alguna actividad de procesamiento. Quedan por realizar estudios funcionales que permitan clarificar si se tratan en algunos casos de pseudoartefactos.

La mayor parte de los artefactos son pequeños y no grandes, como es de esperarse en la mayoría de los contextos arqueológicos de cantera-taller. Esta condición es debido a las características de este tipo de silcrete, que constituye nódulos con un mayor grado de homogeneidad en fragmentos menores a los $10 \mathrm{~cm}$, con respecto a los nódulos de mayor tamaño. Además se pueden relacionar las forma geométricas predominantes registradas en el conjunto lítico analizado (49\% de planas, tabulares, laminares), con las características generales de los nódulos observados en los afloramientos. Estos últimos presentan una mayor continuidad e incluso bandeamiento paralelo a la estratificación de la roca sedimentaria, por lo que la forma geométrica sería una consecuencia del patrón dominante observado en los sedimentos.

\section{Conclusiones}

En este estudio se ha caracterizado una fuente de materia prima, cuya descripción geológica, análisis macroscópico y microscópico han permitido determinar a esta roca

\begin{tabular}{lcc}
\hline Clases artefactuales & $\mathbf{n}$ & $\%$ \\
\hline Núcleos & 10 & 2,53 \\
Lascas & 19 & 4,80 \\
Filos con retoques & 16 & 4,04 \\
Filos con rastros complementarios & 17 & 4,29 \\
Filos con muescas & 3 & 0,76 \\
Ecofactos & 111 & 28,03 \\
Desechos indiferenciados & 219 & 55,30 \\
Bifaces & 1 & 0,25 \\
Total & 396 & 100 \\
\hline
\end{tabular}

Tabla 1. Clases artefactuales

Table 1. Artifact classes

como un silcrete. Los artefactos de esta materia prima han sido empleados por sociedades cazadoras-recolectoras y agroalfareras en el valle, en sitios como El Ranchito y otros del valle de Copacabana.

En El Ranchito se han hallado tres fuentes de aprovisionamiento de este material, conformando áreas de cantera-taller, en la cual una de las muestras dio como resultado la presencia casi el total de artefactos confeccionados en esta materia prima, con un alto porcentaje de ecofactos y desechos de talla, y en menor medida núcleos e instrumentos retocados o filos con rastros complementarios.

Los estudios microscópicos muestran un gran potencial sumado a la caracterización de muestras de mano y a la geología local, por lo que la comparación de muestras de silcrete con otros sitios arqueológicos permitirá identificar relaciones de movilidad con regiones más amplias.

\section{Córdoba, 15 de febrero de 2016}

\section{Agradecimientos}

Este trabajo está siendo desarrollado en el marco de subsidios de Secyt UNC y Foncyt PICT 2013-1029 "Arqueología del Valle de Copacabana, Córdoba: recursos líticos a través del tiempo". Agradecemos a Roxana Cattáneo, Andrés Laguens y Claudio Carignano quienes aportaron información a esta investigación. También a Maximiliano Córdoba, Gabriela Díaz y Lisandro Bigi por los trabajos de campo. A Carolina Mosconi por el abstract. A Florencia Costantino y Soraya Montero por el trabajo de laboratorio. A los evaluadores y editores. Por último a la gente de Chuña por su hospitalidad.

\section{Bibliografía}

Aschero, C. 1975. Ensayo para una clasificación morfológica de artefactos líticos aplicada a estudios tipológicos comparativos. Informe inédito presentado al consejo Nacional de Investigaciones Científicas y Técnicas (CONICET). 
Aschero, C. 1983. Ensayo para una clasificación morfológica de artefactos líticos aplicada a estudios tipológicos comparativos. Revisión. Cátedra de Ergología y Tecnología de la Facultad de Filosofía y Letras de la U.B.A. MS en DIPA, IMHICIHU, CONICET.

Austral, A. \& A. M. Rochietti. 2004. Al sur de Rio Cuarto: síntesis de la arqueología regional. En Bechis, M. (comp). Terceras Jornadas de Arqueología Histórica y de Contacto del Centro Oeste de la Argentina y Seminario de Etnohistoria. Cuartas Jornadas de Arqueología y etnohistoria del Centro Oeste del país. Universidad Nacional de Río Cuarto. Córdoba. Vol. II: 97114. Universidad Nacional de Río Cuarto.

Caminoa, J. M. 2015. “Un estudio de tecnología lítica desde la antropología de las técnicas: el caso del Alero Deodoro Roca ca. 3000 AP, Ongamira, Ischilín, Córdoba". Facultad de Filosofía y Humanidades, Universidad nacional de Córdoba, Argentina, $152 \mathrm{pp}$. Tesis de licenciatura. Ms.

Candiani, J. C., C. Carignano, P. Stuart-Smith, P. Lyons, R. Miró \& H. López. 2001. Hoja Geológica 3166-II. Cruz del Eje, provincias de Córdoba, La Rioja y Catamarca. Boletín № 249. Servicio Geológico Minero Argentino. Buenos Aires.

Carignano, C. 1997. "Caracterización y evolución de los ambientes geomorfológicos extraserranos del noroeste de la Provincia de Córdoba, durante el Cuaternario Superior". Facultad de Ciencias Exactas Físicas y Naturales, Universidad Nacional de Córdoba, Argentina, 207 pp. Tesis Doctoral. Ms.

Cattáneo, G. R. 1994. Estrategias tecnológicas: un modelo aplicado a las ocupaciones prehistóricas del Valle de Copacabana, N.O. de la Provincia de Córdoba. Publicaciones Arqueología 47: 1-30.

Cattáneo, G. R. \& J. Caminoa. 2015. El estudio de conjuntos líticos de cuarzo: el caso del alero Deodoro Roca (Ongamira, Depto Ischilín. Córdoba). Trabajo presentado en las VI Jornadas Arqueológicas Cuyanas. Los Reyunos, San Rafael, Mendoza.

Cerrato Casado, E. 2011. La Prospección Arqueológica Superficial. Un Método No Destructivo para una Ciencia que Sí lo Es. Arte, arqueología e historia 18: 151-160.

Flörke, O.W., H. Gratsch, B. Martin, K. Röller \& R. Wirth. 1991. Nomenclature of microcrystalline and non-crystalline silica minerals, based on structure and microstructure. Neues Jahrbuch Miner ABH 163(1):19-42.

González, A. R. 1956-58. Reconocimiento arqueológico de la zona de Copacabana, Córdoba. Revista do Museu Paulista (N.S), X: 173-223.

González, A. R. 1960. La estratigrafía de la gruta de Intihuasi (Prov. de San Luis, R.A.) y sus relaciones con otros sitios precerámicos de Sudamérica. Revista del Instituto de Antropología (UNC) I: 1-290.

Heider, G. 2015. “Los Pueblos Originarios en el norte de Pampa Seca: una mirada arqueológica a los cazadores recolectores del sur de las provincias de Córdoba y San Luis, Argentina". Facultad de Filosofía y Humanidades, Universidad Nacional de Córdoba, Argentina, 423 pp. Tesis de doctorado. Ms.

Laguens, A. 1993-1994. Observación controlada y análisis estadístico de procesos de formación en un sitio en el árido del centro de Argentina. Relaciones de la sociedad Argentina de Antropología XIX: 215-255.

Laguens, A. 1999. Arqueología del contacto hispano indígena. Un estudio de cambios y continuidades en las Sierras Centrales de Argentina. BAR Internacional Series 801.

Laguens, A., M. Bonnin. 1987. Espacio, paisaje y recursos. Estrategias indígenas alternativas y complementarias en la cuenca del río Copacabana, Sitio El Ranchito. Publicaciones Instituto de Antropología XLV (1): 159-201.

Marcellino, A. 2001. Esqueletos humanos del acerámico en Córdoba: yacimiento de Chuña (sitio El Ranchito), Dpto. Ischilín. Boletín de la Academia Nacional de Ciencias 66: 135-174.

Nash D. \& S. McLaren. 2007. Geochemical sediments and landscapes. D. Nash and S. McLaren (Ed.). 1-465, Blackwell Publishing, United States.

Rivero, D. \& S. Pastor. 2004. Sistemas de producción lítica de las comunidades productoras de alimentos de las sierras de Córdoba. Análisis de tres conjuntos de la pampa de Achala. En Bechis, M. (comp.). Terceras Jornadas de Arqueología Histórica y de Contacto del Centro Oeste de la Argentina y Seminario de Etnohistoria. Cuartas Jornadas de Arqueología y etnohistoria del Centro Oeste del país. Vol. II: 67-80. Universidad Nacional de Río Cuarto, Córdoba.

Sario, G. 2011. "Poblamiento humano en la provincia de San Luis: una perspectiva arqueológica a través del caso de la organización de la tecnología en Estancia La Suiza". Facultad de Filosofía y Humanidades, Universidad Nacional de Córdoba, Argentina, 289pp. Tesis doctoral. Ms.

Sario, G. 2013a. Tecnología lítica en el Valle de Copacabana (Dto. Ischilín, Córdoba En Bárcena, R. y S, Martin (eds.). XVIII Congreso Nacional de Arqueología Argentina, Arqueología Argentina en el Bicentenario de la Asamblea General Constituyente del Año 1813, P.478. Universidad Nacional de La Rioja y CONICET-INCIHUSA. Zeta editores.

Sario, G. 2013b. Sources of lithic material procurement in Estancia La Suiza archeological locality (San Luis, Argentina). Journal of Archaeological and Anthropological Sciences 5(3): 245-254.

Sario, G. \& E. Pautassi. 2015. Canteras-taller de cuarzo y un análisis de los conjuntos artefactuales del sitio Piedra Blanca (Copacabana, Córdoba). Revista Arqueología 21(2): 165-175.

Sario, G., M. Salvatore \& E. Pautassi. 2015. Caracterización de una cantera arqueológica de silcrete en el Valle de Copacabana, Córdoba, Argentina. Trabajo presentado en las $4^{\circ}$ Jornadas de Bienes Culturales. Bariloche, Río Negro. 